\title{
ANÁLISE FATORIAL MULTIVARIADA APLICADA A CARACTERIZAÇÃO DE ÁREAS DE OCORRÊNCIA DE BABAÇU (ATTALEA SPECIOSA MART. ex SPRENG) NA BACIA DO RIO COCAL
}

\section{Mulivariate factor analysis applied to the characterization of Babassu (Attalea Speciosa Mart. ex Spreng) occurrence area in Cocal river basin}

Miriam Rodrigues da Silva Mestre e Colaboradora do Instituto Nacional de Meterologia (INMET) miriamsibmol@gmail.com

Osmar Abilio de Carvalho Júnior Professor Dr. da Universidade de Brasília, Brasília/DF - Brasil osmarjr@unb.br

Éder de Souza Martins

Dr. e Pesquisador A da EMBRAPA Cerrados, Brasília/DF - Brasil eder@cpac.embrapa.br

Danielle Mitja

Dr. do Institut de Recherche pour le Développement - IRD

Danielle.Mitja@ird.fr

Homero Chaib Filho

Dr. e Pesquisador A, EMBRAPA Cerrados, Brasília/DF - Brasil

homero@cpac.embrapa.br

Artigo recebido em 05/10/2011 e aceito para publicação em 08/05/2012

RESUMO: O presente artigo possui como objetivo avaliar a distribuição espacial do babaçu na bacia do rio Cocal, estado de Tocantins. Os dados foram obtidos em levantamento de campo considerando 80 parcelas, considerando os diferentes ambientes presentes na área de estudo. $\mathrm{O}$ estudo considerou 16 variáveis referentes às características do babaçu (quantidade de indivíduos, quantidade de cachos, altura máxima do fuste e altura média do fuste) e do meio ambiente (altitude, declividade, área de contribuição, índice topográfico, relevo, distância do rio, distância das casas, distância da estrada, declividade do relevo no campo, solo, uso do solo e vegetação). A análise dos dados foi realizada utilizando técnicas de sistema de informações geográficas e análise multivariada. O emprego da análise fatorial múltipla permitiu classificar as variáveis ambientais de maior significância para a ocorrência do babaçu e da quantidade de cachos. O principal fator que controla a distribuição do babaçu é o manejo adotado pelos fazendeiros.

Palavras chave: Babaçu, análise fatorial multivariada, dados ambientais, sistema de informação geográfica.

ABSTRACT: This paper aims to evaluate the spatial distribution of the babassu palm in the Cocal River basin, Tocantins State. Data were obtained from field survey considering 80 plots, covering the different environments present in the study area. The research considered 16 variables about the babassu palm characteristics (number of individuals, number of babassu nut clusters, average stem height and maximum stem height) and environment features (elevation, slope, contributing area, topographic index, relief, distance from 
Análise fatorial multivariada aplicada a caracterização de áreas de ocorrência de babaçu (attalea speciosa mart. ex spreng) na bacia do rio Cocal

Miriam Rodrigues da Silva, Osmar Abílio de Carvalho Junior, Eder de Souza Martins, Danielle Mitja, Homero Chaib Filho

the river, distance from the homes, distance from the road, slope obtained in the field, soil, land use and vegetation). Data analysis was performed using techniques of geographic information system and multivariate analysis. The use of multiple factor analysis enabled to classify the environmental variables of greatest significance for the babassu occurrence. The main factor controlling the babassu distribution is the management adopted by farmers.

Keywords: babassu palm, multivariate factor analysis, environmental data, geographic information system.

\section{INTRODUÇÃO}

O Babaçu (Attalea speciosa Mart. ex Spreng) consiste em uma palmeira robusta e imponente com estipe isolado (tronco) de até 20 metros de altura e de 25 a 44 centímetros de diâmetro, com 7 a 22 folhas medindo de 4 a 8 metros de comprimento (SILVA e TASSARA, 1991; HENDERSON, 1995; LORENZI, 1996 et al., BRANDÃO et al., 2002). Ocorre tanto na floresta Amazônica, quanto no bioma Cerrado e sua área estimada de ocorrência no Brasil é de 200.000 $\mathrm{km}^{2}$ (MAY et al., 1985).

Na região nuclear do Cerrado o Babaçu ocorre em áreas restritas, embora localmente possam ocupar áreas extensas. Em geral, os Palmeirais do Cerrado estão em terrenos bem drenados nos interflúvios, e as espécies dominantes pertencem aos gêneros como Acrocomia, Attalea ou Syagrus. Quando o dossel é tipicamente descontínuo ou não há formação de dossel, os palmeirais comumente são formados pelas espécies Acrocomia aculeata (que caracteriza o Macaubal) ou Syagrus oleracea (Guerobal). Em contraposição, quando os palmeirais apresentam dossel contínuo, a espécie dominante é a Attalea speciosa (Babaçu) (RIBEIRO e WALTER, 1998).

O Babaçu pode ocorrer isoladamente nas florestas ou em áreas abertas, sendo mais freqüentemente encontrado em áreas degradadas onde é considerada uma espécie pioneira e dominante. Geralmente, o Babaçu possui baixa densidade na vegetação primária (ANDERSON e MAY 1985; PETERS et al, 1989; ANDERSON et al., 1991). No entanto, o processo de alteração da vegetação, seja por cultivos ou pastagens, pode tanto desencadear a eliminação total ou parcial do Babaçu, como ainda, desenvolver o seu predomínio nestas áreas. Portanto, a presença de Babaçu associa-se fortemente às áreas antropizadas, quando coloniza antigas formações florestais desmatadas (RIBEIRO e WALTER, 1998). Nas áreas de pastagem intensiva o Babaçu, geralmente, é eliminado, enquanto em sistemas mais extensivos o Babaçu, normalmente, é mantido no terreno. Em função do manejo dado pelo produtor rural, o Babaçu pode se encontrar em densidades compatíveis com desenvolvimento das pastagens ou invadir a área. Após 30 anos, a área pode se transformar numa formação secundária quase monoespecífica de Babaçu (MITJA e FERRAZ, 2001). Desta forma, a floresta de Babaçu pode ser considerada como uma formação secundária, que rapidamente se desenvolve nos meses subseqüentes às queimadas, quando seus cocos germinam e as plântulas crescem vigorosamente.

O Babaçu destaca-se como umas das melhores fontes alternativas de energia renovável. Várias partes do coco de Babaçu podem ser utilizadas como fontes energéticas: o mesocarpo para produção de álcool, o endocarpo para produção de carvão e gases, a amêndoa para produção de óleo, com possível aplicação em motores a diesel e o epicarpo para utilização direta como combustível primário (NASCIMENTO, 2004). Além do uso como carvão, o Babaçu vem sendo pesquisado como fonte alternativa para o biodiesel fazendo parte do projeto Nacional Probiodiesel do Ministério da Ciência e Tecnologia, MTC, lançado no ano de 2002 (SANTOS et al., 2006; SILVA et al., 2006; LIMA et al., 2007).

O presente trabalho possui como objetivo caracterizar as áreas de ocorrência do Babaçu na bacia do rio Cocal, situada no município de Natividade no Estado do Tocantins, a partir do mapeamento, levantamento de dados e tratamento das informações utilizando o sistema de informações geográficas e a análise fatorial multivariada.

Soc. \& Nat., Uberlândia, ano 24 n. 2, 267-282, mai/ago. 2012 


\section{MATERIAIS E MÉTODOS}

\section{Levantamento das características do Babaçu e das variáveis ambientais}

No trabalho de campo foram estudadas 80 parcelas em unidades de paisagens típicas do bioma Cerrado: vegetação natural, áreas de pastagens e de uso agrícola (Figura 1). A quantificação da densidade do Babaçu (número de indivíduos/unidade amostral) utilizou áreas de $2500 \mathrm{~m}^{2}$. Pelo fato das superfícies de terreno não serem iguais e para que cada parcela pudesse ter $2500 \mathrm{~m}^{2}$, as medidas foram realizadas das

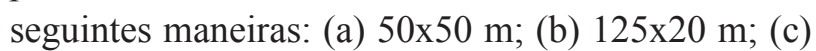
duas parcelas de $50 \times 20$ e uma de $25 \times 20 \mathrm{~m}$; (d) uma parcela de $75 \times 20$ e uma de $50 \times 20 \mathrm{~m}$; e (e) uma parcela de $100 \times 20$ e uma de $25 \times 20 \mathrm{~m}$ (Figura 2). Porém, onde não foi possível no campo foram selecionadas parcelas menores que $2500 \mathrm{~m}^{2}$. Neste caso, foram consideradas as seguintes configurações: uma parcela de $660 \mathrm{~m}^{2}$, duas de $1000 \mathrm{~m}^{2} \mathrm{e}$ cinco de $2000 \mathrm{~m}^{2}$. Posteriormente, os dados obtidos foram transformados em número de indivíduos por hectare. A contagem de indivíduos considerou apenas Babaçus com copa desenvolvida. Dentro de cada parcela, estes Babaçus foram contados e georreferenciados sendo avaliados para todos os indivíduos a altura do fuste e o número de cachos femininos (frutos e flores). Desta forma, foram definidas as seguintes variáveis para análise: quantidade de palmeiras de Babaçu por hectare, quantidade de cachos por hectare, altura máxima do fuste e altura média do fuste.

Além disso, foram levantadas para cada parcela informações sobre as variáveis ambientais (relevo, uso e cobertura da Terra e solo). Em campo foi realizada uma medição da declividade do terreno para cada parcela. As demais informações morfométricas foram extraídos do Modelo Digital de Terreno (MDT) elaborado a partir da interpolação da base cartográfica digital na escala 1:100.000 do Sistema Cartográfico do Tocantins, contendo as curvas de nível, pontos cotados e hidrografia. Os dados foram corrigidos e interpolados pelo módulo TOPOGRID (Hutchinson, 1989) presente no programa ArcInfo. O MDT foi elaborado com uma resolução espacial de 20 metros. A partir do MDT foram gerados os seguintes atributos do terreno: declividade, área de contribuição e índice topográfico. A delimitação das unidades de relevo foi realizada por interpretação visual das informações morfométricas utilizando técnicas de processamento digital de imagens como composição colorida e manipulação de contrastes, que permitem realçar as feições e os padrões do relevo e a posterior análise visual das unidades (SILVA et al 2009).

O mapa de uso de cobertura da Terra foi elaborado a partir do processamento digital de imagem do sensor multiespectral ASTER (Advanced Spaceborne Thermal Emission and Reflection Radiometer). A metodologia adotada apresenta as seguintes etapas: (a) pré-processamento, (b) segmentação, (c) classificação não-supervisionada, e (d) edição e geração do mapa de vegetação (CARVALHO JUNIOR et al, 2005, SILVA et al. 2009). As classes obtidas foram: (a) Mata de Galeria, (b) Mata Seca, (c) Formações Savânicas (Cerrado sentido restrito, Parque Cerrado, Palmeiral e Vereda), (d) Formações Campestres (Campo Sujo, Campo Rupestre e Campo Limpo), (e) Áreas com afloramento de calcário mostrando exposição da rocha com feições pontiagudas, (f) áreas antrópicas referentes a plantio e pastagens, e (g) Mata seca secundária. Tanto a validação do mapa de fisionomias e as ocorrências do babaçu foram confirmadas com o trabalho de campo.

As informações pedológicas foram obtidas a partir do trabalho realizado pelo Estado (TOCANTINS, 2005). Complementarmente, para as parcelas coletadas foi realizada uma caracterização do tipo de solo. As classes de solo predominante na área são: Latossolo Vermelho-Amarelo, Argissolo Vermelho-Amarelo, Neossolo Litólico e Plintossolo Pétrico.

A partir do SIG foram calculadas as variáveis relativas à distância da parcela em relação ao rio, casa e estrada. Todas as informações foram configuradas espacialmente dentro de um SIG contendo para as 80 parcelas as informações das 16 variáveis relativas aos atributos ambientais e estruturais das palmeiras. 
Análise fatorial multivariada aplicada a caracterização de áreas de ocorrência de babaçu (attalea speciosa mart. ex spreng) na bacia do rio Cocal

Miriam Rodrigues da Silva, Osmar Abílio de Carvalho Junior, Eder de Souza Martins, Danielle Mitja, Homero Chaib Filho

Figura 1 - Localização das áreas amostradas no campo.

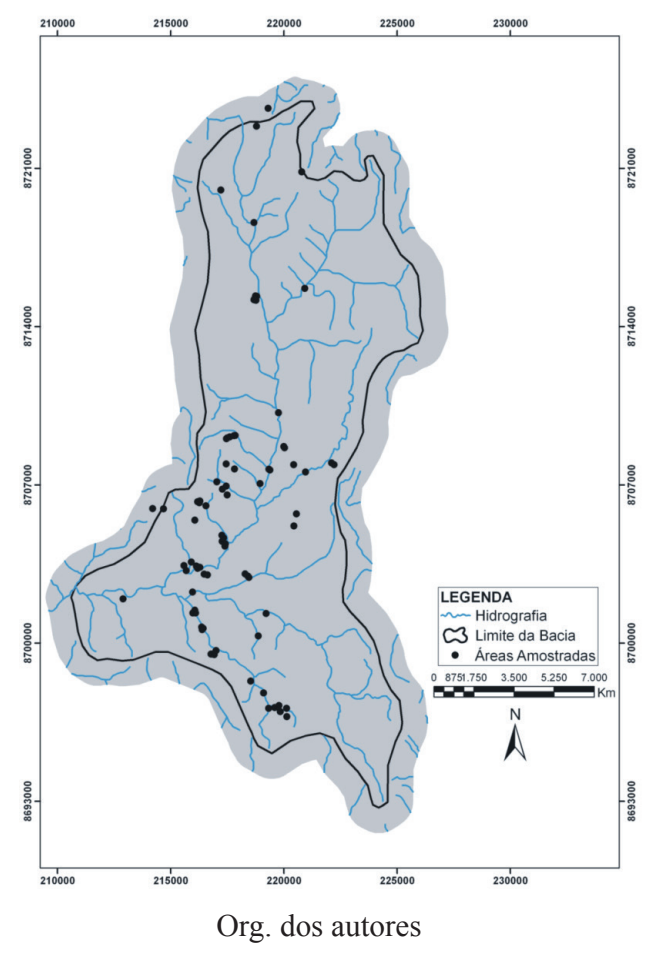

Figura 2 - Configuração das parcelas de campo

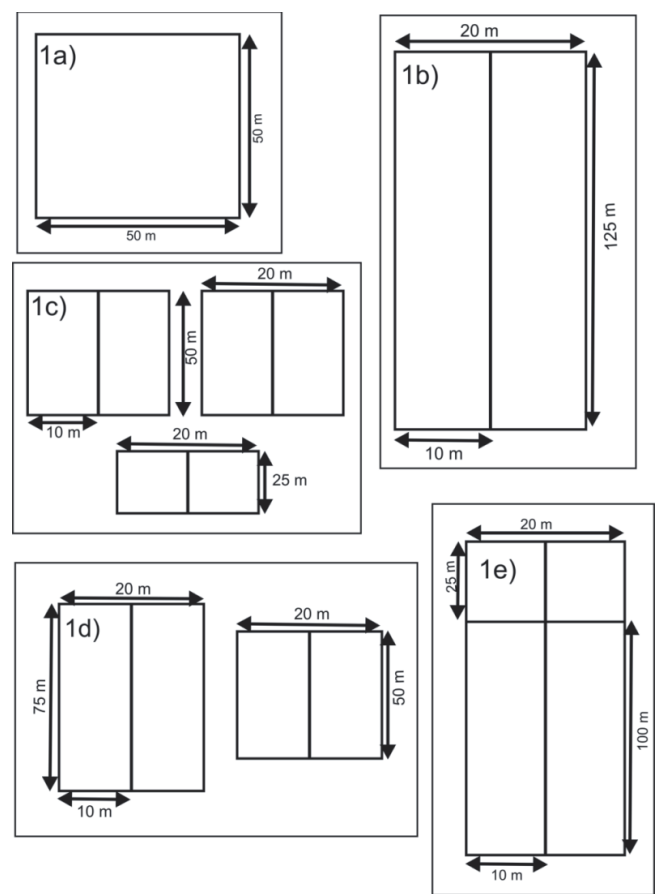

(a) $50 \times 50 \mathrm{~m}$; (b) 125x20 m; (c) duas parcelas de $50 \times 20$ e uma de $25 \times 20 \mathrm{~m}$; (d) uma parcela de $75 \times 20$ e uma de 50x20m; e (e) uma parcela de 100x20 e uma de $25 \times 20 \mathrm{~m}$. Org. dos autores. 


\section{Análise Multivariada}

O emprego de técnicas estatísticas multivariadas é utilizado para compreender as relações, semelhanças ou diferenças entre as características do Babaçu e o seu ambiente. Dentre essas técnicas destaca-se a Análise Fatorial Múltipla (AFM) por reduzir o número de variáveis e evidenciar possíveis agrupamentos de acordo com seu grau de similaridade ou dissimilaridade (ANDRADE, 1989; BOUROCHE e SAPORTA, 1980; ESCOFIER e PAGĖS, 1998; LEBART et al., 2002).

A AFM é um procedimento estatístico, cujo principal objetivo é ordenar e reduzir o número de variáveis correlacionadas entre si, permitindo a explicação da variabilidade do conjunto de dados iniciais (ANDRADE, 1989). As técnicas de AFM têm sido empregadas em diferentes áreas do conhecimento como, por exemplo: análise ambiental (SILVA e RIBEIRO, 2004), qualidade da água (BRITO et al., 2006), análises de solos (GROBE e MARQUES, 2006), estudo de espécies vegetais (DIAS et al., 2007), entre outros. No presente estudo a AFM auxilia na seleção das variáveis com maior contribuição na ocorrência e variabilidade da densidade do Babaçu ou aquelas que indicam a ausência dessa palmeira. A análise fatorial múltipla foi realizada com auxílio do programa LISA 3.0 que foi desenvolvido para integrar variáveis qualitativas e quantitativas e do SAS 9.1 que é um programa integrado para análise de dados estatísticos cuja funcionalidade foi construída em torno de quatro idéias básicas: acesso, administração, análise e apresentação de dados (ESCOFIER e PAGÈS, 1998; LEBART et al., 2002).

A análise multivariada foi realizada de duas formas: (a) considerando o total de parcelas levantadas, e (b) restringido a análise apenas as parcelas contendo Babaçu que totaliza 58 parcelas.

\section{RESULTADOS}

\section{AFM aplicada ao conjunto total de parcelas}

A análise fatorial múltipla mista (AFM) aplicada ao conjunto total de parcelas (80) e variáveis (16) busca caracterizar os atributos ambientais que descrevem a presença do Babaçu. Nesta análise, os quatro primeiros fatores explicam cerca de $60 \%$ da variância total, sendo que: o Fator 1 (F1) explica 24,86\% dessa variância, o Fator 2 (F2) 14,09\%, do Fator 3 (F3) 11,37\% e o Fator 4 (F4) explica 9,32\% (Figura 3). A Tabela 1 apresenta as contribuições das variáveis dos quatro primeiros fatores.

Figura 3 - Gráfico de barras de distribuição percentual da variância representada por cada autovalor.

\begin{tabular}{|c|c|c|c|c|c|}
\hline Fatores & Auto-Valor & Percentagem & $\begin{array}{l}\text { Percentual } \\
\text { Acumulado }\end{array}$ & Variação & \\
\hline 1 & 2.619529 & 24.866 & 24.866 & $* * * * * * *$ & $* * * * * * * * * * * * * * * * * * * * *$ \\
\hline 2 & 1.485035 & 14.097 & 38.963 & 10.769 & $* * * * * * * * * * * * * * * *$ \\
\hline 3 & 1.198282 & 11.375 & 50.337 & 2.722 & $* * * * * * * * * * * * * *$ \\
\hline 4 & 981880 & 9.321 & 59.658 & 2.054 & $* * * * * * * * * * * * *$ \\
\hline 5 & .888466 & 8.434 & 68.092 & .887 & $* * * * * * * * * * * *$ \\
\hline 6 & .750310 & 7.122 & 75.214 & 1.311 & $* * * * * * * * * *$ \\
\hline 7 & .679884 & 6.454 & 81.668 & .669 & $* * * * * * * *$ \\
\hline 8 & .495557 & 4.704 & 86.372 & 1.750 & $* * * * * *$ \\
\hline 9 & .313508 & 2.976 & 89.348 & 1.728 & $* * * * *$ \\
\hline 10 & .251645 & 2.389 & 91.737 & .587 & $* * * * *$ \\
\hline 11 & .195864 & 1.859 & 93.596 & .529 & $* * * *$ \\
\hline 12 & .159542 & 1.514 & 95.110 & .345 & $* * * *$ \\
\hline 13 & .153456 & 1.457 & 96.567 & .058 & $* * * *$ \\
\hline 14 & .098911 & .939 & 97.506 & .518 & $* * *$ \\
\hline 15 & .083374 & .791 & 98.297 & .147 & $* * *$ \\
\hline 16 & .053603 & .509 & 98.806 & .283 & $* *$ \\
\hline 17 & .039644 & .376 & 99.182 & .133 & $* *$ \\
\hline 18 & .028798 & .273 & 99.456 & .103 & $* *$ \\
\hline 19 & .025432 & .241 & 99.697 & .032 & $* *$ \\
\hline 20 & .022326 & .212 & 99.909 & .029 & $* *$ \\
\hline 21 & .009568 & .091 & 100.000 & .121 & $*$ \\
\hline
\end{tabular}


Análise fatorial multivariada aplicada a caracterização de áreas de ocorrência de babaçu (attalea speciosa mart. ex spreng) na bacia do rio Cocal

Miriam Rodrigues da Silva, Osmar Abílio de Carvalho Junior, Eder de Souza Martins, Danielle Mitja, Homero Chaib Filho

As variáveis que mais contribuíram para a formação do primeiro fator $\mathrm{F} 1$ estão presentes na Figura $4 \mathrm{a}$. Observa-se que com sinal negativo ficaram aglutinadas as variáveis correlacionadas com a presença do Babaçu: altura máxima do fuste (AMFU), altura média do fuste (AMEF) e quantidade de palmeiras de Babaçu/ha (QBHA). Em contraposição, com sinal positivo ficaram os fatores inibidores da presença do Babaçu: patamar cárstico (PCAR), distância de rio (DRIO), altitude do Modelo Digital de Terreno (AMDT) e Latossolo Vermelho (LVER). Em outras palavras, a densidade de Babaçu, a altura média e máxima do fuste diminui quando a distância do rio e altitude aumenta especialmente nos Latossolos Vermelhos e no patamar cárstico.

Para a formação do segundo fator F2, as variáveis que mais contribuíram estão presentes no gráfico da Figura 4b. A principal modalidade de variável com sinal negativo é a PCAR, que se refere às parcelas localizadas em áreas planas, com solos profundos e com o estabelecimento de vegetação arbórea. Em contraposição com sinal positivo no fator $\mathrm{F} 2$ estão as variáveis: índice topográfico (TOPG) e declividade (DECL), e as modalidades de variáveis: Neossolo Litólico (NLIT), Argissolo Vermelho (AVER) e forte ondulado (FOND) (Tabela 1). Essas parcelas estão sobre ambientes de re- levo fortemente ondulado caracterizadas por possuírem maior declividade e conseqüentemente maior índice topográfico (que significa menor saturação de água) onde desenvolvem Neossolo Litólico na parte de maior declive ou Argiloso Vermelho nas baixadas.

Para a formação do $\mathrm{F} 3$, as variáveis que mais contribuíram estão no gráfico da Figura 4c. As principais modalidades de variáveis com sinal negativo são: plano (PLAN), Neossolo Flúvico (NFLU), Argissolo Vermelho (AVER), Gleissolo Háplico (GHAP) e Mata de Galeria (uso e cobertura) (USMG). Essas parcelas estão em relevo plano caracterizadas por ambientes de Mata de Galeria degradada onde se desenvolve o Neossolo Flúvico quando ocupa pequenas porções de várzea, Argissolo Vermelho quando os solos são mais profundos e com drenagem moderada ou Gleissolo Háplico quando estão em ambiente hidromórfico sujeito a inundações.

Em contraposição com sinal positivo no fator $\mathrm{F} 3$ está a variável distância do rio (DRIO) e as modalidades de variáveis: Plintossolo Pétrico (PPET), ondulado (ONDL), e Cerrado degradado (UCCE) ou vegetação original (CERR). Os dados indicam que essas parcelas estão em relevo ondulado, distantes do rio, onde se desenvolve o Plintossolo Pétrico e vegetação de Cerrado.

Figura 4. Variáveis de maior contribuição: (a) F1, (b) F2, (c) F3 e (d) F4.
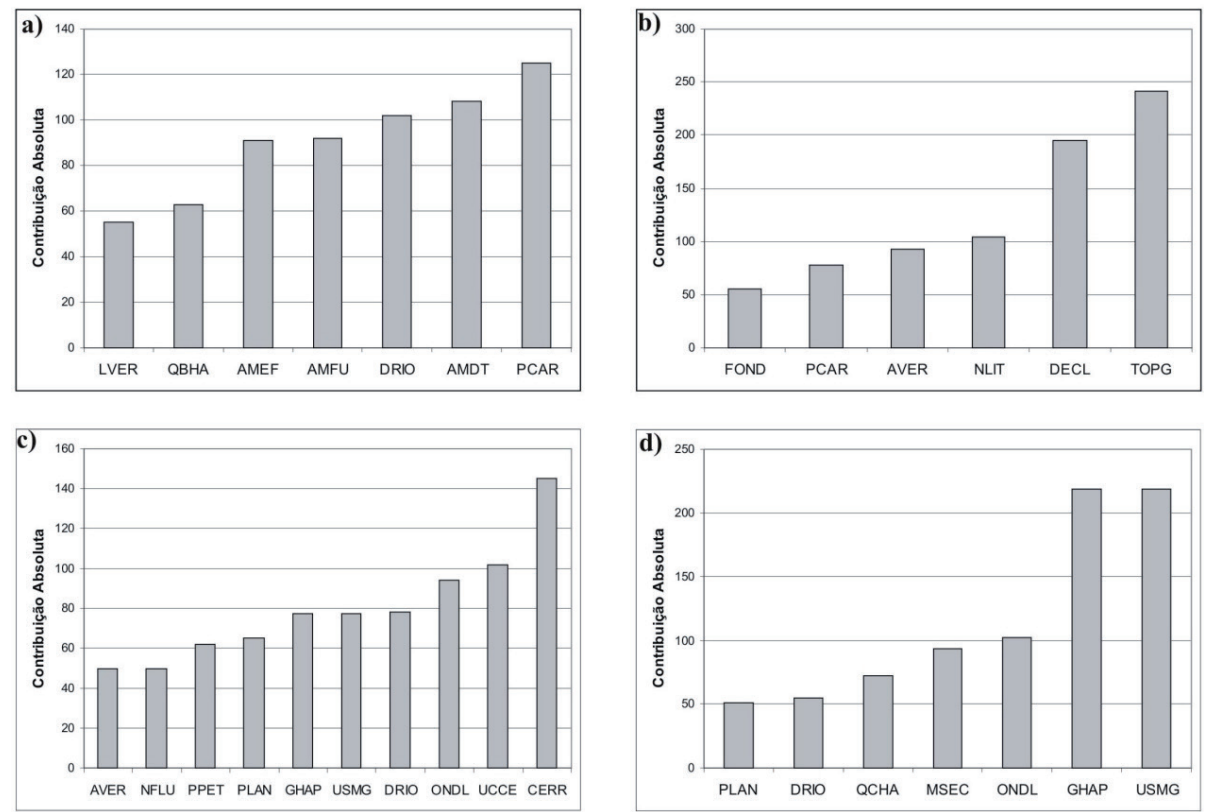

Org. dos autores.

Soc. \& Nat., Uberlândia, ano 24 n. 2, 267-282, mai/ago. 2012 
Análise fatorial multivariada aplicada a caracterização de áreas de ocorrência de babaçu (attalea speciosa mart. ex spreng) na bacia do rio Cocal

Miriam Rodrigues da Silva, Osmar Abílio de Carvalho Junior, Eder de Souza Martins, Danielle Mitja, Homero Chaib Filho

Tabela 1 - Valor das contribuições absoluta e relativa das variáveis aos fatores (x1000-1).

\begin{tabular}{|c|c|c|c|c|c|c|c|c|c|c|c|c|c|c|}
\hline & VAR & QLT & $F_{1}$ & $\begin{array}{l}\mathrm{CO} \\
\mathrm{R}_{1} \\
\end{array}$ & CTR & $F_{2}$ & $\begin{array}{l}\mathrm{CO} \\
\mathrm{R}_{2} \\
\end{array}$ & $\begin{array}{c}\text { CTR } \\
2 \\
\end{array}$ & $F_{3}$ & $\begin{array}{c}\mathrm{CO} \\
\mathrm{R}_{3} \\
\end{array}$ & \begin{tabular}{|c|c|} 
CTR \\
3 \\
\end{tabular} & $\mathbf{F}_{4}$ & $\begin{array}{c}\mathrm{COR} \\
4\end{array}$ & \begin{tabular}{|c|c|} 
CTR \\
4 \\
\end{tabular} \\
\hline & 2H & 551 & -709 & 503 & 63 & -125 & 15 & 2 & 3 & 0 & 0 & 180 & 32 & 10 \\
\hline & 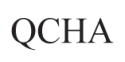 & 389 & -405 & 164 & 20 & 10 & 0 & 0 & -106 & 11 & 3 & 462 & 213 & 72 \\
\hline & MFU & 769 & -855 & 731 & 92 & -69 & 4 & 1 & -34 & 1 & 0 & 178 & 31 & 10 \\
\hline & MEF & 762 & -851 & 724 & 91 & -112 & 12 & 2 & -7 & 0 & 0 & 158 & 25 & 0 \\
\hline & $\mathrm{CL}$ & 835 & 402 & 162 & 31 & 753 & 568 & 195 & -254 & 64 & 27 & -201 & 40 & 21 \\
\hline & MDT & 576 & 744 & 555 & 108 & -117 & 13 & 4 & -87 & 7 & 3 & -7 & 0 & 0 \\
\hline & TOPG & 814 & 214 & 46 & 9 & 837 & 701 & 241 & -258 & 66 & 28 & 4 & 0 & 0 \\
\hline & DRIO & 815 & 724 & 525 & 102 & -3 & 0 & 0 & 429 & 184 & 78 & 326 & 106 & 55 \\
\hline \multirow{3}{*}{ 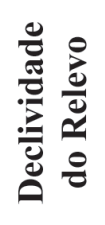 } & AN & 78 & 495 & 245 & 33 & -417 & 174 & 41 & 70 & 221 & 65 & 376 & 141 & 51 \\
\hline & ONDL & 521 & -307 & 94 & 18 & 58 & 3 & 1 & 472 & 223 & 94 & -446 & 199 & 102 \\
\hline & FOND & 238 & -256 & 65 & 12 & 409 & 168 & 55 & 68 & 4 & 1 & 12 & 0 & 0 \\
\hline \multirow{2}{*}{\begin{tabular}{l}
0 \\
\multirow{2}{*}{}
\end{tabular}} & LN & 808 & -769 & 591 & 26 & 458 & 210 & 16 & 21 & 0 & 0 & -79 & 6 & 0 \\
\hline & PCAR & 808 & 769 & 591 & 125 & -458 & 210 & 78 & -21 & 0 & 0 & 79 & 6 & 3 \\
\hline \multirow{7}{*}{ 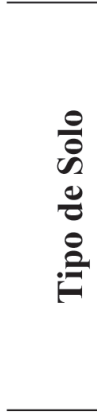 } & 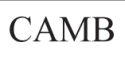 & & -542 & & 27 & & . & 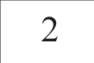 & & 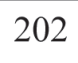 & 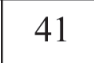 & 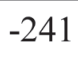 & & $T$ \\
\hline & LVER & 483 & 605 & 366 & 55 & -281 & 79 & 21 & 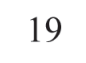 & 0 & 0 & 192 & 37 & 15 \\
\hline & NFLU & 422 & -287 & 82 & 11 & -255 & 65 & 15 & -415 & 172 & 50 & 319 & 102 & 36 \\
\hline & NLIT & 390 & 189 & 35 & 6 & 590 & 348 & 104 & 54 & 3 & 1 & -52 & 2 & 1 \\
\hline & GHAP & 82 & 275 & 75 & 13 & -271 & 73 & 22 & -450 & 202 & 77 & -687 & 472 & 219 \\
\hline & PPET & 344 & 362 & 131 & 22 & -180 & 32 & 9 & 405 & 164 & 62 & 123 & 15 & 7 \\
\hline & AVER & 505 & 245 & 60 & 10 & 551 & 304 & 93 & -365 & 133 & 50 & 82 & 6 & 3 \\
\hline \multirow{4}{*}{ 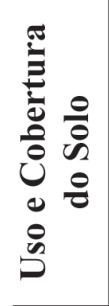 } & 101 & 20 & -396 & 157 & 23 & -142 & 20 & $J$ & -9 & , & 2 & -141 & 20 & 7 \\
\hline & USMG & 824 & 275 & 75 & 13 & -271 & 73 & 22 & -450 & 202 & 77 & -687 & 472 & 219 \\
\hline & PAST & 199 & -101 & 10 & 0 & 116 & 13 & 1 & -120 & 14 & 1 & 400 & 160 & 22 \\
\hline & UCCE & 58 & 514 & 264 & 42 & 142 & 20 & 5 & 539 & 290 & 102 & -75 & 5 & 2 \\
\hline \multirow{3}{*}{ 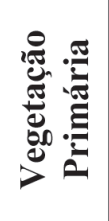 } & CERR & 78 & & 62 & 1 & 9 & 9 & 1 & 7 & 572 & 145 & -243 & 59 & 18 \\
\hline & MGAL & 567 & -439 & 192 & 16 & -326 & 106 & 15 & -511 & 261 & 48 & -81 & 6 & 1 \\
\hline & MSEC & 543 & 303 & 92 & 14 & 352 & 124 & 34 & -319 & 101 & 34 & 474 & 224 & 93 \\
\hline
\end{tabular}

Org. dos autores

Fn - valor da coordenada da variável no enésimo fator ou eixo; VAR - variáveis; QLT - Qualidade de representação total para os fatores considerados ( $\sum$ CORn); CORn - contribuição relativa (ou qualidade de representação) ao fator n; CTRn - contribuição absoluta para a formação do fator n. 
Análise fatorial multivariada aplicada a caracterização de áreas de ocorrência de babaçu (attalea speciosa mart. ex spreng) na bacia do rio Cocal

Miriam Rodrigues da Silva, Osmar Abílio de Carvalho Junior, Eder de Souza Martins, Danielle Mitja, Homero Chaib Filho

As variáveis que mais contribuíram para a formação do quarto fator F4 estão presentes na Figura $4 \mathrm{~d}$. As principais variáveis com sinal positivo são: quantidade de cachos/ha (QCHA) e distância de rio (DRIO), e as modalidades de variáveis são: Plano (PLAN), Mata Seca (MSEC) e Gleissolo Háplico (GHAP). E com sinal negativo foram as seguintes modalidades de variáveis: ondulado (ONDL) e Mata de Galeria degradada (USMG). Isso significa dizer que nessas parcelas a densidade de cacho/ha aumenta quando a distância do rio aumenta, e a vegetação primária aberta é a Mata Seca, enquanto essa densidade é menor nas formações vegetais atuais de Mata de Galeria.

Na Figura 5 estão demonstrados os gráficos de dispersão tanto para as variáveis como para as parcelas entre o primeiro fator (F1) no eixo da abscissa e o segundo fator (F2) no eixo das coordenadas. A análise conjunta dos gráficos é realizada a partir da associação das parcelas e variáveis posicionadas nos mesmos quadrantes nos gráficos de dispersão.

$\mathrm{O}$ gráfico de dispersão mostra à esquerda as parcelas com maior densidade de Babaçu/ha, com exceção da parcela 13, e à direita do gráfico as parcelas que não contem ou possui baixa densidade de Babaçu/ha.

É possível ver a distribuição de cada parcela na Figura 6, que mostra as parcelas com a presença de Babaçu e sem Babaçu. Observa-se que as parcelas com presença de Babaçu estão ligadas a rede de drenagem e as parcelas sem Babaçu estão distantes da drenagem e na cabeceira da bacia.

\section{AFM aplicada ao conjunto de parcelas contendo Babaçu}

A partir dos resultados obtidos pela primeira AFM foi realizado um segundo processamento dos dados considerando apenas as parcelas contendo a presença de Babaçu. A Figura 5, com exceção da parcela 13 que se encontra a direita do gráfico, totalizando 58 parcelas. A partir dessa análise foi possível observar a formação de novos grupos contendo parcelas semelhantes (Figura 7). Esses grupos apresentam-se distribuídos dentro de um arranjo espacial na bacia (Figura 8).
-O grupo 1 possui sete parcelas (P014, $\mathrm{P} 015$, P017, P019, PO20, P023 e P025). Estas parcelas são caracterizadas pela grande quantidade de Babaçu/ha, pelas maiores alturas de fuste, estão localizadas em áreas planas ou suave ondulado, estão próximas do rio em ambientes de Neossolo Flúvico ou Plintossolo Háplico, apresentam uma Mata de Galeria degradada em nível de recuperação (capoeira antiga) e estão distantes da casas.

-O grupo 2 possui sete parcelas (P007, $\mathrm{P} 009$, P018, P024, P034, P043 e P044). Este grupo é caracterizado pela quantidade de Babaçu/ha e altura máxima de fuste, está localizado em ambiente de relevo plano a suave ondulado, consequentemente próximo dos rios e em locais onde se desenvolve o Neossolo Flúvico, também está em áreas de Mata de Galeria degradada e em processo de recuperação (capoeira antiga) e distantes das casas.

-O grupo 3 possui sete parcelas (P012, P016, P046, P047, P051, P058, e P060). A principal característica desse grupo é que está em ambiente de vegetação primária de Mata de Galeria, apresenta um relevo colinoso, a área de contribuição possui valores baixos, estão localizados em ambientes de cerca de 300 metros de distância da estrada. Estas parcelas também se caracterizam pela quantidade de Babaçu/ha e altura máxima de fuste, e estão em locais de relevo plano ou suave ondulado.

-O grupo 4 possui cinco parcelas (P006, P011, $\mathrm{P} 022$, P048 e P059). Este grupo distinguiu-se principalmente por está em locais cuja vegetação primária é a Mata de Galeria com certo nível de recuperação (capoeira nova ou antiga), em ambientes de colinas e próximo da estrada. Mostra uma área de contribuição com valores baixos, consequentemente está em relevo plano. Também apresentam uma grande quantidade de Babaçu/ha com uma altura máxima de fuste.

-O grupo 5 possui cinco parcelas (P049, P052, P053, P054 e P055). Estão em ambientes de Mata de Galeria degradada e próxima dos rios. Encontram-se a cerca de 250 metros de distância da estrada, em locais

Soc. \& Nat., Uberlândia, ano 24 n. 2, 267-282, mai/ago. 2012 
de relevo colinoso onde desenvolve o Cambissolo e possui valores médios a altos de área de contribuição.

-O grupo 6 possui seis parcelas (P013, $\mathrm{P} 035$, P036, P037, P038 e P39). São parcelas que possuem uma grande quantidade de cachos/ha e as maiores médias de altura de fuste. Estão caracterizadas por apresentarem um ambiente de Mata Seca ou de transição de Mata de Galeria para Cerrado, estão próximas do rio e com altitude com cerca de 370 metros, e em locais com declividade média de 3,5 graus, com exceção da parcela 13.

-O grupo 7 possui uma única parcela (P021) com características ambientais singulares. O Babaçual encontra-se próximo de um córrego, estando apenas separado por um terraço hidromórfico com solo pálido e com blocos de canga laterítica. Provavelmente, o presente ambiente está correlacionado com a dinâmica fluvial, onde o córrego sofreu um deslocamento do seu leito mantendo os Babaçus em um fragmento de cerrado.

-O grupo 8 também possui uma única parcela (P057). Apresenta uma baixa densidade de Babaçu/ ha (48 indivíduos), porém uma grande quantidade de cachos/ha (144 cachos). E ainda se encontra em um local cultivado por pastagem o que pode explicar a quantidade de cachos de Babaçu.

-O grupo 9 possui sete parcelas (P026, P027, P028, P031, P032, P042 e P062). A principal característica desse grupo é de pertencer a um ambiente de vegetação primária de Cerrado, em locais de relevo ondulado a forte ondulado onde se desenvolve o Cambissolo e predomina as pastagens.

-O grupo 10 possui sete parcelas (P040, P041, P045, P050, P061, P063 e P064). A principal característica de deste grupo é está localizado em ambientes de relevo forte ondulado onde consequentemente se desenvolve o Cambissolo e se encontram em locais de roças ou pastagens.
- O grupo 11 possui cinco parcelas (P029, P030, P033, P056 e P065). O que diferencia este grupo do grupo 10 é que está em ambientes de relevo ondulado a forte ondulado.

A partir dos resultados obtidos observa-se que a análise fatorial múltipla permitiu classificar as variáveis de maior significância para a ocorrência de densidade de Babaçu e densidade de cachos na área amostrada. De modo geral, ocorreram variações significativas nas variáveis analisadas, indicando um comportamento diferenciado na presença e densidade do Babaçu e também um comportamento diferenciado para formação de cada grupo. Normalmente, os locais de maior densidade de palmeiras não são os locais de maior densidade de cacho. A maior densidade de cachos está associada às áreas cultivadas. Esses resultados mostraram também que a densidade de palmeiras e de cachos está relacionada a ambientes que possuem Cambissolo e Neossolo Flúvico. Os resultados permitem dizer que o fator que mais influencia a densidade do Babaçu é o tipo de manejo aplicado pelo agricultor. Dependendo do tipo de manejo a diversidade e a concentração do Babaçu na bacia é eliminada ou aumentada. 
Análise fatorial multivariada aplicada a caracterização de áreas de ocorrência de babaçu

(attalea speciosa mart. ex spreng) na bacia do rio Cocal

Miriam Rodrigues da Silva, Osmar Abílio de Carvalho Junior, Eder de Souza Martins, Danielle Mitja, Homero Chaib Filho

Figura 5. Representação das variáveis, das parcelas no primeiro plano fatorial considerando todas as parcelas amostradas.

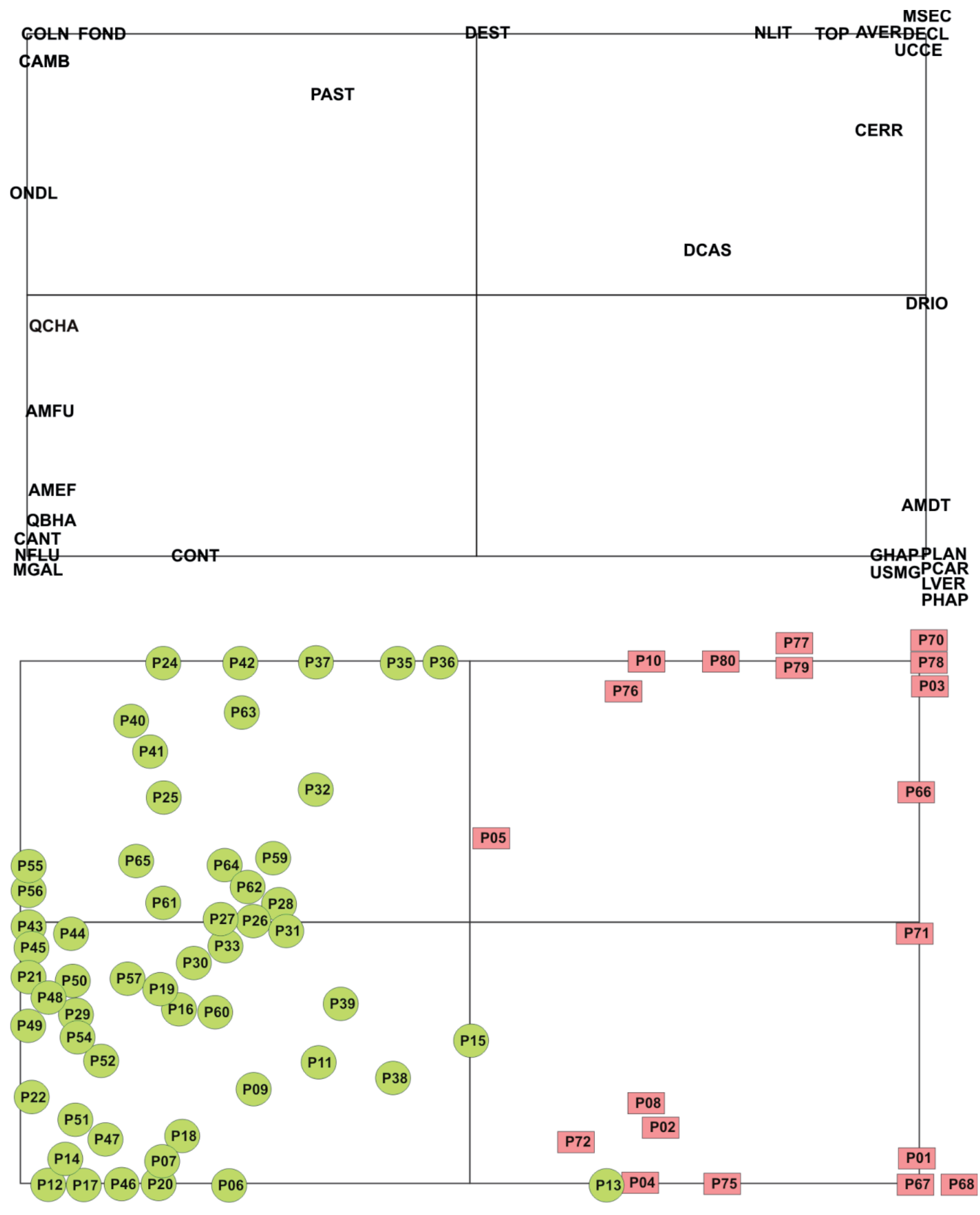

Org. dos autores.

Soc. \& Nat., Uberlândia, ano 24 n. 2, 267-282, mai/ago. 2012 
Análise fatorial multivariada aplicada a caracterização de áreas de ocorrência de babaçu (attalea speciosa mart. ex spreng) na bacia do rio Cocal Miriam Rodrigues da Silva, Osmar Abilio de Carvalho Junior, Eder de Souza Martins, Danielle Mitja, Homero Chaib Filho

Figura 6. Localização das parcelas com e sem presença de Babaçu na bacia

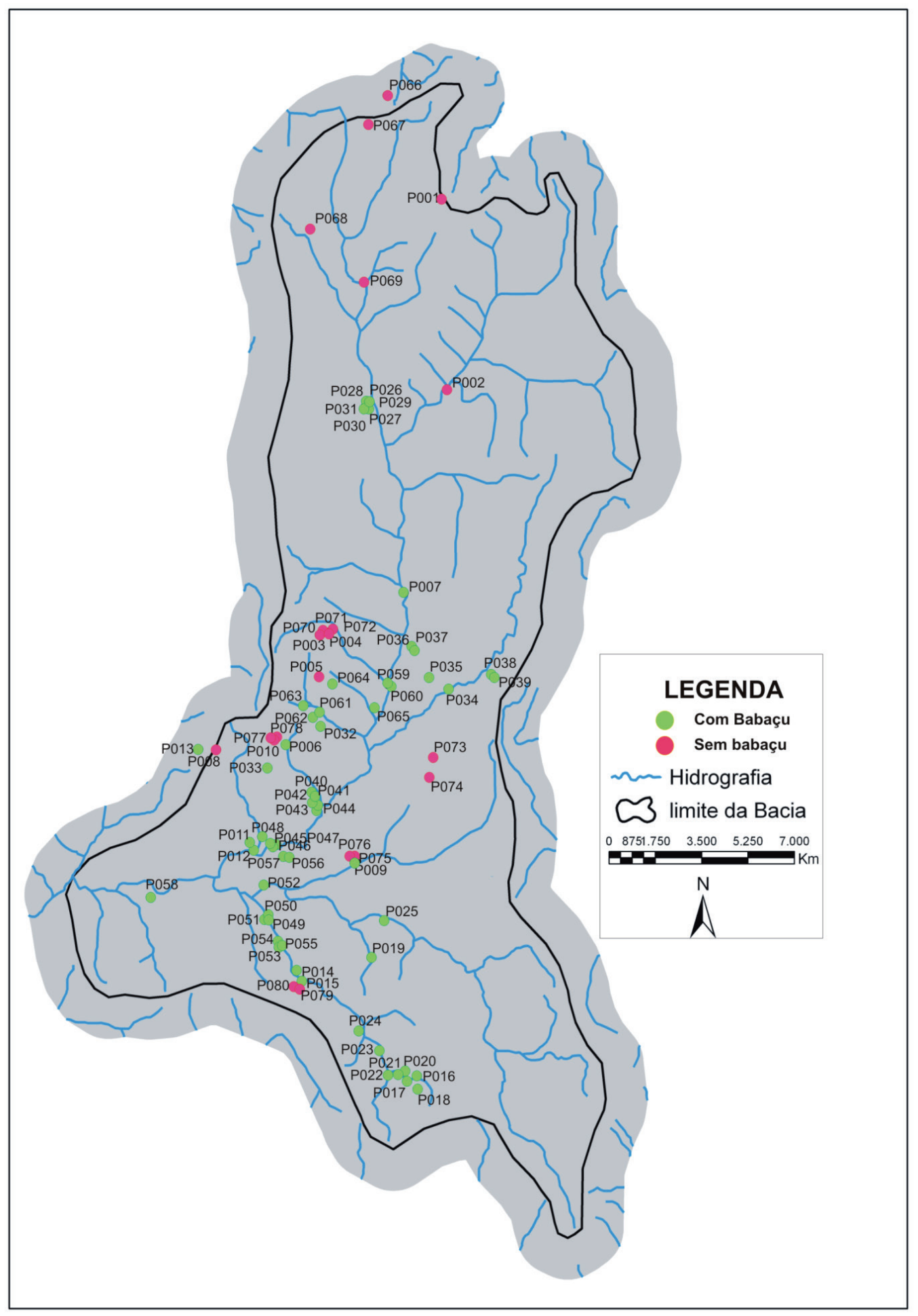

Org. dos autores. 
Análise fatorial multivariada aplicada a caracterização de áreas de ocorrência de babaçu (attalea speciosa mart. ex spreng) na bacia do rio Cocal

Miriam Rodrigues da Silva, Osmar Abilio de Carvalho Junior, Eder de Souza Martins, Danielle Mitja, Homero Chaib Filho

Figura 7. Representação das variáveis e das parcelas com Babaçu no primeiro plano fatorial considerando as parcelas com presença de babaçu.
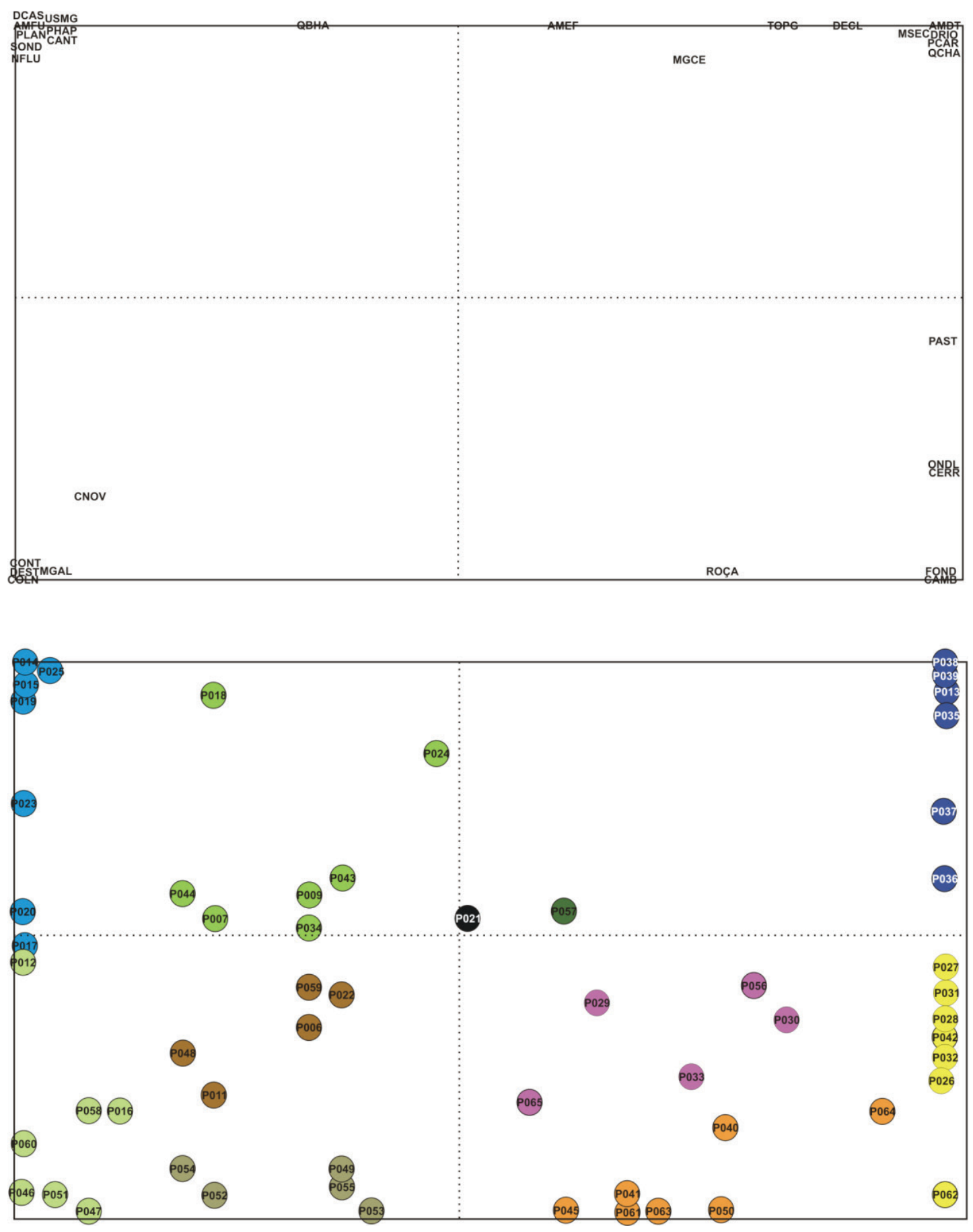

Org. dos autores.

Soc. \& Nat., Uberlândia, ano 24 n. 2, 267-282, mai/ago. 2012 
Análise fatorial multivariada aplicada a caracterização de áreas de ocorrência de babaçu (attalea speciosa mart. ex spreng) na bacia do rio Cocal

Miriam Rodrigues da Silva, Osmar Abilio de Carvalho Junior, Eder de Souza Martins, Danielle Mitja, Homero Chaib Filho

Figura 8 - Localização dos grupos na bacia obtidos pela análise fatorial a partir das parcelas com presença de babaçu.

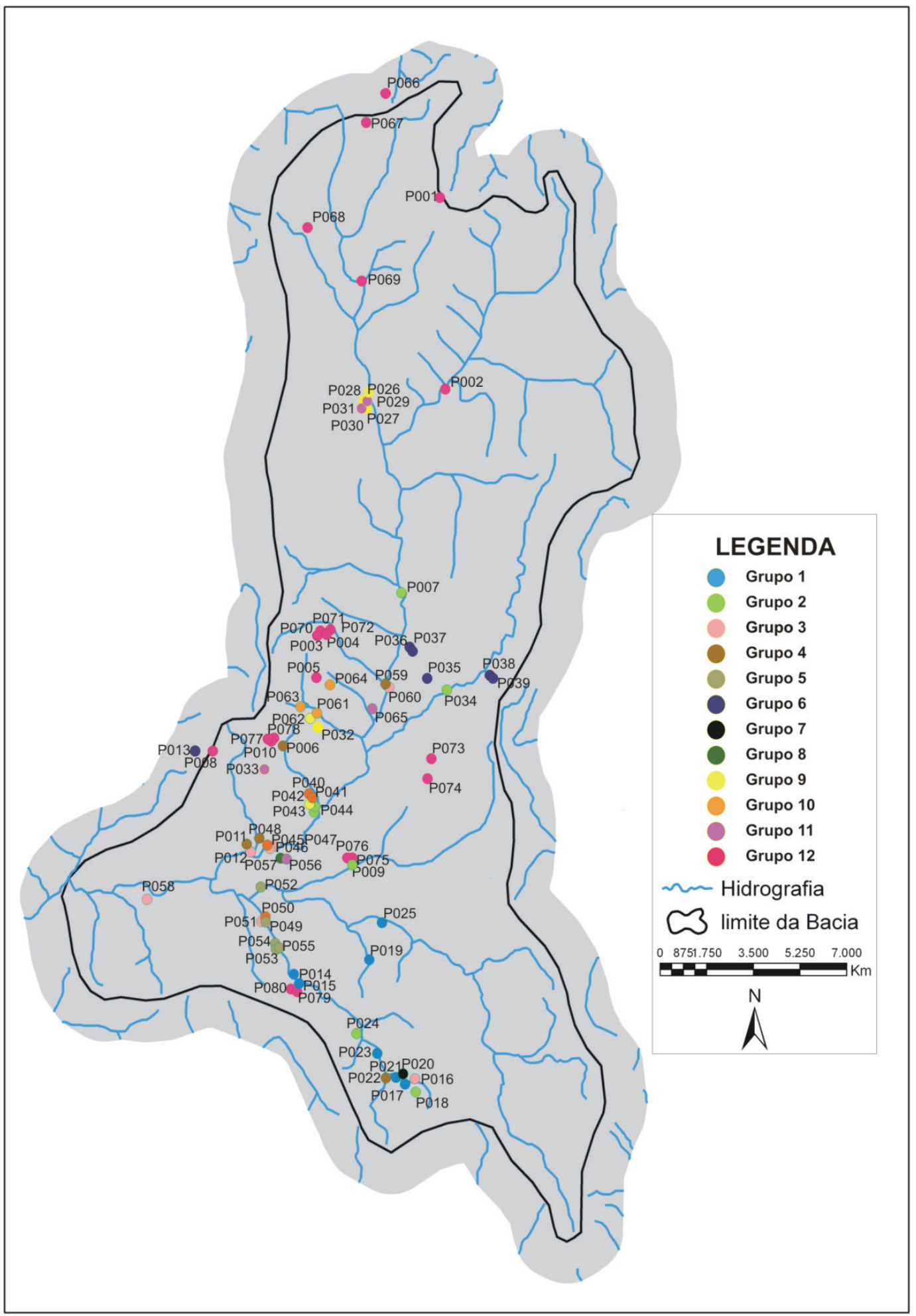

A classe 12 foi incluída de forma a representa as parcelas sem babaçu identificadas na análise fatorial anterior presente na FIG.6 e 7. Org. dos autores. 
Análise fatorial multivariada aplicada a caracterização de áreas de ocorrência de babaçu (attalea speciosa mart. ex spreng) na bacia do rio Cocal

Miriam Rodrigues da Silva, Osmar Abílio de Carvalho Junior, Eder de Souza Martins, Danielle Mitja, Homero Chaib Filho

\section{CONCLUSÕES}

O emprego da análise fatorial múltipla conseguiu reduzir um grande número de informações ambientais e estruturais, a um pequeno número de fatores que sintetizam a caracterização das áreas de ocorrências do Babaçu na bacia do rio Cocal, sendo estas representadas pelas Matas de Galeria, Mata Seca secundária, Colinas e áreas de pastagens e roças, além de permitir e classificar as variáveis de maior significância para a ocorrência de densidade de Babaçu e densidade de cachos na área de estudo, além de indicar um comportamento diferenciado para formação dos grupos.

Os procedimentos empregados podem ser seguidos e testados em outras áreas de Babaçus podendo servir como uma ferramenta auxiliar na análise de variáveis que contribuem para a presença ou ausência da espécie.

\section{REFERÊNCIAS}

ANDERSON, A. B.; MAY, P. H.; BALICK, M. J. The subsidy from nature: Palm Forests, Peasantry and development on an Amazonian Frontier. Nova York: Columbia University Press, 1991. 230 p.

ANDERSON, A.; MAY, P. A palmeira de muitas vidas. Ciência Hoje, 1985, v.4, n. 20, p. 58-64.

ANDRADE, T.A. Métodos estatísticos e econométricos aplicados à análise regional. In: HADDAD, P.R.; FERREIRA, C.M. de C.; BOISIER, S.; ANDRADE, T.A. (Ed.). Economia regional: teorias e métodos de análise. Fortaleza: BNB-ETENE, 1989. p.427-507.

BOUROCHE, J.M.; SAPORTA, G. Análise de dados. Tradução de: M. Penchel. Rio de Janeiro: Zahar Editores, $1980.116 \mathrm{p}$.

BRANDAO, M.; LACA-BUENDIA, J. P.; MACEDO, J. F. Árvores nativas e exóticas do estado de Minas Gerais. Belo Horizonte: EPAMIG, 2002. 528 p.

BRITO, L. T. L.; SILVA, A. S.; SRINIVASAN, V. S; GALVÃO, C. O.; GHEYI, H. R. Uso de análise multivariada na classificação das fontes hídricas subterrâneas da bacia hidrográfica do Salitre. Engenharia Agrícola, Jaboticabal, SP, v. 26, n. 1, p. 36-44, 2006.

CARVALHO JUNIOR, O. A.; GUIMARÃES, R. F.; MARTINS, E. S.; CARVALHO, A. P. F.; GOMES, R. A. T. Aplicação do método de identificação espectral para imagens do sensor ASTER em ambiente de cerrado. Revista Brasileira de Geofísica, v. 23, p. 159-172, 2005.

DIAS, P. F.; SOUTO, S. M.; COSTA, J. R. Análise do comportamento de espécies leguminosas arbóreas introduzidas em pastagens de gramíneas tropicais. Pesquisa Agropecuária Tropical (UFG), v. 37, p. 31-37, 2007.

ESCOFIER, B.; PAGÈS, J. Analyse factorialles simples et multiples: Objectifs, méthodes et interpréta-

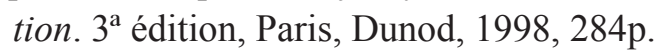

GROBE, J. R.; MARQUES, J. M. O método de análise fatorial aplicado ao estudo de resultados de análise de solos. Synergismus scyentifica UTFPR, v. 1, p. 237-243, 2006.

HENDERSON, A. The palms of the Amazon. New York: Oxford University Press, 1995. 362 p.

HUTCHINSON, M.F. (1989). A new procedure for gridding elevation and stream line data with automatic removal of spurious pits. Journal of Hydrology, 106: 211-232.

LEBART, L.; MORINEAU, A.; PIRON, M. Statistique exploratoire multidimensionnelle. $3^{\mathrm{a}}$ ed. Nouveau tirage révisé Paris: Dunod, 2002, 439 p.

LIMA, J. R. O.; SILVA, R. B.; SILVA, C. C. M.; SANTOS, L. S. S.; SANTOS JUNIOR, J. R.; MOURA, E. M.; MOURA, C. V. R. Biodiesel de babaçu (Orbignya sp.) obtido por via etanólica. Química Nova, v. 30, n. 3, 600-603, 2007.

LORENZI, H.; SOUZA, H.M., de; MEDEIROS-COSTA, J.T. de; CERQUEIRA, L.S.C. de; BEHR, N. von. Palmeiras no Brasil: nativas e exoticas. Nova Odessa: Plantarum, 1996. 303p.

Soc. \& Nat., Uberlândia, ano 24 n. 2, 267-282, mai/ago. 2012 
MAY, P. H.; ANDERSON, A. B.; FRAZÃO, J. M. F.; BALICK, M. J. Babassu palm in the agroforestry systems in Brazil's Mid-North region. Agroforestry Systems, v. 3, p. 275-295, 1985.

MITJA, D.; FERRAZ, I. D. K. Establishment of babassu in pastures in Pará, Brazil. Palms, v. 45, n. 3, p. 138-147, 2001.

NASCIMENTO, U. S. Carvão de babaçu como fonte térmica para sistema de refrigeração por absorção no estado do Maranhão. 2004. 82p. Dissertação (Mestrado em Engenharia Mecânica) - Faculdade de Engenharia Mecânica (FEM) - Universidade Estadual de Campinas.

PETERS, C.; BALICK, M. J.; KAHN, F.; ANDERSON, A. Oligarchic florests od economic plants in Amazonia: Utilization and conservation of an important tropical resource. Conservation Biology, v. 3, n. 4, p. $342-349.1989$.

RIBEIRO, J.F.; WALTER, R. M. T. Fitofisionomia do bioma Cerrado. In: SANO, S. M.; ALMEIDA, S. P. de (Eds.). Cerrado: ambiente e flora. Planaltina: EMBRAPA- CPAC, 1998. p. 89-166.

SILVA, M. R.; CARVALHO JUNIOR, O. A.; MARTINS, E. S.; MITJA, DANIELLE, GOMES, R. A. T. \& GUIMARÃES, R. F. (2009). Mapeamento de áreas potenciais do babaçu (ATTALEA SPECIOSA MART. EX SPRENG) na bacia do rio Cocal, Tocantins. Espaço e Geografia, v.12, n.1, p. 1-29, 2009.

SILVA, R. G.; RIBEIRO, C. G. Análise da degradação ambiental na Amazônia Ocidental: um estudo de caso dos municípios do Acre. Revista de Economia e Sociologia Rural, v. 42, p. 93-112, 2004.

SILVA, S.; TASSARA, H. Frutas no Brasil. São Paulo: Empresa das Artes, 1991. 230p.

TOCANTINS (Estado). Secretaria do Planejamento e Meio Ambiente. Diretoria de Zoneamento Ecológico-econômico. Atlas do Tocantins: subsídios ao planejamento da gestão territorial. Palmas: SEPLAN, 2005. 54p. 